\title{
Hereditäre Netzhautdystrophien aufgrund von RPE65-Varianten: Von der genetischen Diagnostik zur Therapie
}

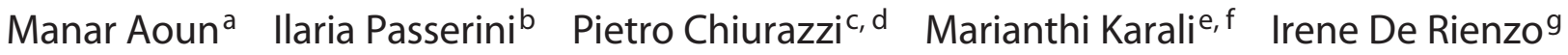 \\ Giovanna Sartor $^{\text {h }}$ Vittoria Murro ${ }^{i}$ Natalia Filimonova ${ }^{j}$ Marco Seri ${ }^{\text {h, }}{ }^{\text {S Sandro Banfi }}{ }^{\mathrm{f}, \mathrm{I}}$

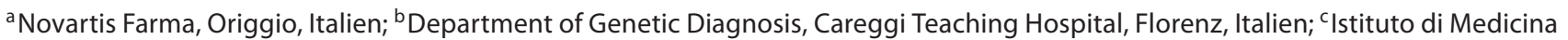 \\ Genomica, Università Cattolica del Sacro Cuore, Rom, Italien; ${ }^{\mathrm{d}}$ Fondazione Policlinico Universitario «A. Gemelli» IRCCS, UOC Genetica \\ Medica, Rom, Italien; ${ }^{\circledR}$ Eye Clinic, Multidisciplinary Department of Medical, Surgical and Dental Sciences, Università degli Studi della \\ Campania «Luigi Vanvitelli», Neapel, Italien; ${ }^{\mathrm{f}}$ Telethon Institute of Genetics and Medicine (TIGEM), Pozzuoli, Italien; ${ }^{9}$ Department of \\ Ophthalmology, AOU-Careggi, Florenz, Italien; hedical Genetics Unit, IRCCS Azienda Ospedaliero-Universitaria di Bologna, Bologna, \\ Italien; 'Department of Neuroscience, Psychology, Drug Research and Child Health, University of Florence, Eye Clinic Careggi Teaching \\ Hospital, Florenz, Italien; ${ }^{j}$ Novartis Pharma AG, Basel, Schweiz; ${ }^{k}$ Department of Surgical and Medical Sciences, University of Bologna, \\ Bologna, Italien; 'Medical Genetics, Department of Precision Medicine, University of Campania «Luigi Vanvitelli», Neapel, Italien
}

\section{Schlüsselwörter \\ hereditäre Netzhautdystrophie $\cdot$ RPE65 · Next-Generation- \\ Sequenzierung · Variante unklarer Signifikanz · Gentest . \\ genetische Beratung}

\section{Zusammenfassung}

Die hereditären Netzhautdystrophien (IRD; inherited retinal diseases) sind eine heterogene Gruppe von Krankheiten - unter anderem Retinitis pigmentosa (RP), Lebersche kongenitale Amaurose (LCA) und Netzhautdystrophie mit frühem Krankheitsbeginn (EO[S]RD; earlyonset [severe] retinal dystrophy) -, die sich hinsichtlich der Schwere der Krankheit und des Alters bei Krankheitsbeginn unterscheiden. IRD können durch Mutationen in > 250 verschiedenen Genen hervorgerufen werden. Varianten des RPE65-Gens sind die Ursache für 0,6-6\% aller Fälle von RP und für 3-16\% der Fälle von LCA/EORD. Voretigen Neparvovec ist eine Gentherapie, die zur Behandlung von Patienten mit autosomal-rezessiver Retinadystrophie infolge biallelischer RPE65-Varianten (RPE65-IRD) zugelassen ist. Die korrekte molekulare Diagnostik ist daher bei RPE65-IRD von entscheidender Be- deutung, um therapeutisch nutzbare, d. h. potenziell von der Therapie profitierende Genotypen zu identifizieren, und sie ist eine tragende Säule des Patientenmanagements. Es sind bereits Hunderte von RPE65-Varianten bekannt, und während ein Teil als pathogen oder wahrscheinlich pathogen identifiziert werden konnte, ist die Signifikanz anderer Varianten noch unklar. In dieser Übersichtsarbeit beschreiben wir das erforderliche gendiagnostische Vorgehen, um diejenigen Patienten auszuwählen, die die Voraussetzungen für die Behandlung mit Voretigen Neparvovec erfüllen. Die sorgfältige klinische Charakterisierung der Patienten durch interdisziplinäre Expertenteams in Kombination mit der Verfügbarkeit von Next-Generation-Sequenzierung kann den Zugang der Patienten zu verfügbaren Therapieoptionen beschleunigen. (c) 2021 Die Autoren
Marco Seri und Sandro Banfi haben zu gleichen Teilen zur Erstellung dieser Arbeit beigetragen.
Korrespondenz an:

Sandro Banfi, banfi@tigem.it 


\section{Einleitung}

Die hereditären Netzhautdystrophien (IRD; inherited retinal diseases) sind eine Gruppe von Krankheiten, die mit fortschreitendem Verlust des Sehvermögens einhergehen und zusammengenommen eine Prävalenz zwischen 1:3000 und 1:4000 haben $[1,2]$. Die verschiedenen IRD haben unterschiedliche genetische Ursachen und phänotypische Ausprägungen $[1,2]$. Retinitis pigmentosa (RP) ist eine der häufigsten IRD [3]. Bei RP geht der Verlust von Photorezeptoren primär in der Netzhautperipherie mit dem Auftreten von Pigmentablagerungen, den so genannten Knochenkörperchen, einher $[4,5]$. Je nach Lebensalter bei Krankheitsbeginn, Schweregrad, Progressionsgeschwindigkeit und Erscheinungsbild sind die häufigsten IRD, die mit starker Sehbeeinträchtigung schon im Kindesalter einhergehen, die Lebersche kongenitale Amaurose (LCA) und die früh einsetzende schwere Netzhautdystrophie (EO[S]RD; early-onset [severe] retinal dystrophy) mit folgenden Merkmalen: erheblich eingeschränktes Sehvermögen ab der Geburt oder dem frühen Säuglingsalter, «wandernder» Nystagmus, amaurotische Pupillen sowie deutlich reduzierte oder nicht erfassbare Ganzfeld-Elektroretinogramme [6, 7].

Mutationen in mehr als 250 verschiedenen Genen sind bisher mit IRD in Verbindung gebracht worden $[1,2,8]$. Zu diesen potenziell für IRD verantwortlichen Genen zählen Genvarianten von RPE65, die RP (RP20, OMIM \# 613794) und LCA/EORD (LCA2, OMIM \# 204100) verursachen können [7, 9, 10].

RPE65-IRD zeigen variable natürliche Verläufe und treten typischerweise zwischen der Geburt und dem fünften Lebensjahr erstmals auf $[11,12]$. Patienten mit RPE65-bedingten IRD zeigen in der Regel ein deutlich verändertes Sehverhalten («Lichtsuchen» bei ausgeprägter Nachtblindheit, Nystagmus) [7], stark verminderte bis nicht-nachweisbare Fundusautofluoreszenz bis $488 \mathrm{Na}$ nometer bei relativ unauffälligem Aussehen des Fundus [13], nicht-vorhandenes Elektroretinogramm oder residuelle $30-\mathrm{Hz}$ Flimmerreaktion [14] sowie schwach hypermetrope oder myope Refraktionsfehler [10]. Einige Patienten haben in jungen Jahren zwar noch eine normale oder annähernd normale Sehschärfe (Visus), doch auch sie erleiden erhebliche Einbußen im Laufe des ersten Lebensjahrzehnts. Im Alter von 15 bis 20 Jahren beginnt sich der Visus zu verschlechtern, und nach dem 20. Lebensjahr beschleunigt sich dieser Prozess. Bei vielen Patienten verschlechtert sich der Visus bis hin zur Blindheit im Sinne des Gesetzes (Visus $=20 / 200$ ) im Alter von 16 Jahren [12]. Im vierten Jahrzehnt waren alle Patienten, über die hier berichtet wird, erblindet im Sinne des Gesetzes, und viele hatten keinerlei Lichtwahrnehmung (vollständiger Verlust des Sehvermögens). Weniger schwere Ausprägungen der Krankheit sind bei Personen mit hypomorphen RPE65-Allelen beschrieben worden [15-18]. Insbesondere wurden ungewöhnliche phänotypische Manifestationen von biallelischen RPE65-Mutationen bei vier Familien mit Albipunctatusähnlichen Fundusveränderungen sowie einer Familie mit starker Hyperopie festgestellt [19].

In der großen Mehrheit der Fälle handelt es sich bei RPE65-IRD um autosomal-rezessiv vererbte Erkrankungen. Aber auch ein autosomal-dominantes Vererbungsmuster wird von einigen Autoren bei einem kleinen Teil der Fälle diskutiert [20-22].

2017 erhielt die Gentherapie Voretigen Neparvovec (Luxturna ${ }^{\circledR}$, Spark Therapeutics, Philadelphia, PA, USA) von der US-Aufsichtsbehörde Food and Drug Administration die Zulassung zur Behandlung von Patienten mit Netzhautdystrophie aufgrund einer nachgewiesenen biallelischen RPE65-Mutation und mit lebensfähigen Netzhautzellen [23]. Ein Jahr später wurde Voretigene Neparvovec von der Europäischen Arzneimittelagentur in einer ähnlichen Indikation zugelassen, nämlich zur Behandlung von erwachsenen und pädiatrischen Patienten mit Sehverlust aufgrund einer erblichen Netzhautdystrophie, die auf nachgewiesenen biallelischen RPE65-Mutationen beruht, und die über ausreichend lebensfähige Netzhautzellen verfügen [24].

Das Ziel dieser Arbeit ist es, eine Übersicht über die Evidenz zu den genetischen Grundlagen der RPE65-IRD zu geben, mit Schwerpunkt auf den bestgeeigneten Ansätzen für die molekulare Testung von Patienten, die mögliche Kandidaten für eine RPE65-Gensupplementierung sind.

\section{Epidemiologie der RPE65-IRD}

Schätzungen zufolge leiden weltweit mehr als 2,5 Millionen Menschen unter einer IRD [25]; die häufigste Form ist die RP mit einer Prävalenz von 1:4000 [3, 26, 27]. Der Anteil der RP unter den IRDPatienten wird mit $21 \%$ bis $54 \%$ beziffert $[28,29]$. Die Prävalenz von LCA/EORD hingegen liegt zwischen 1:30 000 und 1:80 000 $[10,30]$. Auf LCA/EORD entfallen etwa $5 \%$ aller IRD; die berichteten Zahlen reichen von 2,59\% bis 22\% [28-30].

Eine korrekte Einschätzung der Prävalenzraten der RPE65-Mutationen wird dadurch erschwert, dass frühere Studien unterschiedliche Patientenpopulationen, Erstdiagnosen und Beurteilungsmethoden betrachteten und ein gemeinsamer Nenner fehlt (Tabelle 1).

RPE65-Varianten sind für $0,8-1,5 \%$ aller Fälle von IRD verantwortlich $[34,39,40]$. Für die USA wurde die Häufigkeit von RPE65-Mutationen auf 1:576 667 Personen geschätzt; das entspricht einer Gesamtzahl von 563 betroffenen Personen pro Zeitpunkt oder 7 neuen Fällen pro Jahr [32]. Eine Analyse von Genotyp-Daten aus sechs großen Weltpopulationen ergab die Vorhersage, dass es weltweit 15620 Personen mit biallelischen RPE65-Mutationen und dass mehr als 60\% davon aus der afrikanischen Bevölkerung stammen [33].

RPE65-Mutationen bildeten je nach untersuchter Kohorte die Grundlage von etwa 0,6-6\% der RP-Fälle und 3-16\% der LCA/ EORD-Fälle [9, 10, 19, 29, 31, 36-38, 41, 42]. Die größte europäische Studie zur Epidemiologie der IRD, in der die genetische Landschaft einer Kohorte von 6089 IRD-Patienten (4403 Familien) in Spanien analysiert wurde, ergab RPE65-Mutationen bei 3\% der Familien mit autosomal-rezessiver RP [38]. In einer italienischen Studie wurden RPE65-Varianten bei 8,4\% der Patienten mit LCA/EORD identifiziert [31] - ein höherer Wert als die veröffentlichte Prävalenz von RPE65-Mutationen bei Patienten mit LCA/ EORD in nordwesteuropäischen Ländern $(1,7-6,1 \%)[6,43]$. Der
116

Kompass Ophthalmol 2021;7:115-123 DOI: $10.1159 / 000519136$ 
Tab. 1. Epidemiologie der Leberschen kongenitalen Amaurose, der Retinitis pigmentosa und RPE65-bedingter erblicher Netzhauterkrankungen

\begin{tabular}{|c|c|c|}
\hline Studie & Teilnehmer* & Ergebnisse* \\
\hline Simonelli et al. 2007 [31] & $\begin{array}{l}\text { Italienische Patienten mit LCA/EORD } \\
N=95\end{array}$ & RPE65-Mutationen in der Stichprobe: $n=8(8,4 \%)$ \\
\hline Stone et al. 2017 [32] & $\begin{array}{l}\text { Konsekutive IRD-Patienten eines einzelnen Arztes } \\
N=1^{\prime} 000\end{array}$ & $\begin{array}{l}\text { RPE65-Mutationen in der Stichprobe: } n=3 \\
\text { Geschätzte Häufigkeit in der US-Bevölkerung: 1:576 } 667\end{array}$ \\
\hline Avela et al. 2019 [28] & $\begin{array}{l}\text { Finnische Kinder mit IRD } \\
N=68\end{array}$ & $\begin{array}{l}\text { RP: } n=14(21 \%) \\
\text { LCA/EORD: } n=15(22 \%)\end{array}$ \\
\hline Chung et al. 2019 [12] & $\begin{array}{l}\text { Patienten mit IRD aufgrund biallelischer RPE65-Mutationen } \\
N=70\end{array}$ & $\begin{array}{l}\text { LCA/EORD: } n=39(50,0 \%) \\
\mathrm{RP}: n=6(7,7 \%)\end{array}$ \\
\hline Hanany et al. 2020 [33] & Genotyp-Daten zu sechs großen Weltpopulationen & $\begin{array}{l}\text { Autosomal-rezessive IRD: } 1: 1380 \\
\text { RP: } 23 \% \\
\text { LCA/EORD: } 7 \% \\
\text { RPE65 biallelisch: } n=15620 \text { Personen oder 0,3\% aller } \\
\text { IRD-Patienten }\end{array}$ \\
\hline Holtan et al. 2020 [29] & $\begin{array}{l}\text { Norwegische Patienten mit IRD; } N=866 \\
n=685 \text { im Südosten Norwegens lebend }\end{array}$ & $\begin{array}{l}\text { RP: } n=468(54,0 \%) \\
\text { LCA/EORD: } n=45(5,2 \%) \\
\text { RPE65-bedingte RP und LCA/EORD: 0,6\% } \\
\text { Minimale adjustierte IRD-Prävalenz in Südost-Norwegen: } \\
\text { 1:3856 }\end{array}$ \\
\hline Pontikos et al. 2020 [34] & $\begin{array}{l}\text { Patienten mit IRD } \\
\text { Personen: } N=4^{\prime} 236\end{array}$ & $\begin{array}{l}\text { RPE65: } n=51(1,2 \%) \\
\text { LCA/EORD: } 3 \%\end{array}$ \\
\hline Sharon et al. 2020 [35] & $\begin{array}{l}\text { Israelische Patienten mit IRD } \\
\text { Personen: } N=3^{\prime} 413\end{array}$ & $\begin{array}{l}\text { RP: } 43 \% \\
\text { LCA/EORD: } 4 \% \\
\text { RPE65: } 1 \% \text { der IRD-Patienten }\end{array}$ \\
\hline Whelan et al. 2020 [36] & $\begin{array}{l}\text { Irische Patienten mit IRD } \\
N=1^{\prime} 004\end{array}$ & $\begin{array}{l}\text { RP: } 37,75 \% \\
\text { RPE65: } 7,41 \% \text { der autosomal-dominanten RP } \\
\text { LCA/EORD: } 2,59 \% \\
\text { RPE65: 6,25\% der LCA/EORD }\end{array}$ \\
\hline Colombo et al. (2021) [37] & $\begin{array}{l}\text { Italienische Patienten mit RP } \\
N=591\end{array}$ & $\begin{array}{l}\text { AR-RP: } 103 / 591(17,5 \%) \\
\text { RPE65: 2/103 (2\%) der AR-RP }\end{array}$ \\
\hline Perea-Romero et al. (2021) [38] & $\begin{array}{l}\text { Spanische Patienten mit IRD } \\
N=66^{\prime} 089 \text { Personen (4'403 Familien) }\end{array}$ & $\begin{array}{l}\text { Nicht-syndromale RP: } 55,6 \% \text { der Familien } \\
\text { RPE65: 23/666 (3\%) der Familien mit AR-RP }\end{array}$ \\
\hline
\end{tabular}

AR-RP: autosomal-rezessiv vererbte Retinitis pigmentosa; EORD: early-onset retinal dystrophy (Netzhautdystrophie mit frühem Krankheitsbeginn); IRD: inherited retinal disease (hereditäre Netzhautdystrophie); LCA: Lebersche kongenitale Amaurose; RP: Retinitis pigmentosa. * Die Studien wurden an heterogenen Populationen mit unterschiedlichen Ausgangsdiagnosen und mit unterschiedlichen Fragestellungen durchgeführt.

Grund hierfür könnte sein, dass in der italienischen Studie die Patienten nach klinischen Merkmalen einer RPE65-assoziierten Erkrankung, nämlich der Diagnose LCA/EORD, vorselektiert wurden. Und tatsächlich wurden in einer später durchgeführten italienische Studie RPE65-Mutationen bei 3,4\% der IRD-Patienten identifiziert [41], und die neueste Publikation beziffert die Prävalenz von RPE65-Mutationen bei Patienten mit autosomalrezessiver RP mit 2\% [37]. Den Erfahrungen der Autoren mit einem sehr großen Patientenkollektiv aus dem ganzen Land liegen biallelische RPE65-Mutationen etwa bei 1-2\% aller in Italien zur Gentestung überwiesenen IRD-Patienten vor; jährlich werden 4-5 neue Fälle identifiziert (unveröffentlichte Daten). Kürzlich führte das European Vision Institute Clinical Research Network (EVICR.net) eine multinationale Erhebung durch, unter anderem um etwas über die Verteilung von RPE65-IRD in Europa zu erfahren. In dieser Erhebung gaben drei Viertel der befragten itali- enischen Zentren an, 1-5 Patienten mit RPE65-IRD in Behandlung zu haben; bei einem Viertel waren es 6-10 Patienten [44].

\section{Molekularbiologie von RPE65}

Beim Menschen liegt das RPE65-Gen auf Chromosom 1 (1p31) und ist über $20 \mathrm{~kb}$ lang [26]. RPE65 umfasst 14 Exons und codiert für das retinale-Pigmentepithel-spezifische $65 \mathrm{kDa}$-Protein (RPE65) [45, 46], dessen Bezeichnung laut aktueller Nomenklatur Retinoid-Isomerohydrolase RPE65 lautet. RPE65 besteht aus 533 Aminosäuren [45]. Es ist ein hoch konserviertes Protein, das in großen Mengen ausschließlich im retinalen Pigmentepithel (RPE) exprimiert wird [45]. Es gibt zwei Formen von RPE65 - eine membranständige Form (mRPE65) als Hauptform mit Palmitoylierung und eine lösliche Form (sRPE65) [45, 47]. RPE65 ist am Sehzyklus beteiligt, einem mehrphasigen Prozess, bei dem das ins 
Auge einfallende Licht in elektrische Signale umgewandelt wird, die dann an das Gehirn weitergeleitet werden. Wenn photosensitive Pigmente in der Netzhaut von Licht getroffen werden, wandeln sie 11-cis -Retinal in All-trans-Retinal um. RPE65 ist eine Isomerase, die all-trans-Retinylester wiederum in 11-cis-Retinol umwandelt, das dann für die erneute Photoisomerisierung bereit ist [45]. Ein Mangel an RPE65 führt zu einer verminderten 11-cisRetinol-Konzentration und zur Akkumulation von Retinylestern im RPE [45].

\section{Sequenzvarianten des RPE65-Gens im Überblick}

Eine Gentherapie kommt nur für Patienten mit biallelischer Mutation und mit lebensfähigen Photorezeptorzellen in Frage. Die Durchführung der Gentherapie bei RPE65-IRD umfasst eine bilaterale Vitrektomie, gefolgt von einer subretinalen Injektion [48]. Wegen der hiermit verbundenen Risiken ist es wichtig eindeutig festzustellen, wie pathogen die zugrunde liegenden Mutationen sind und ob beide Allele betroffen sind. Die genetische Diagnostik ist von zentraler Bedeutung, um sicherzustellen, dass die Patienten die Voraussetzung für die Behandlung erfüllen [49]. Eine wichtige Aufgabe der Gendiagnostik bei IRD besteht darin, diejenigen Genotypen zu identifizieren, die als pathogen und damit als therapeutisch nutzbar einzustufen sind (die also wahrscheinlich auf die zugelassene Gensupplementierung ansprechen werden - analog zum Konzept der therapeutisch nutzbaren Mutationen bei Krebserkrankungen, für die es zielgerichtete Therapieoptionen gibt) [50]. Da es sehr viele komplexe und unklare Varianten gibt, ist ein umfassendes genetisches Screening umso wichtiger.

\section{Varianten}

Ein Zusammenhang zwischen RPE65-Varianten und LCA/EORD wurde erstmals 1997 hergestellt [51, 52]. Die Heterogenität der vorgefundenen Varianten zeigte sich sofort - Gu et al. berichteten über fünf verschiedene Varianten (eine Missense-Mutation [p.Pro363Thr], zwei Punktmutationen, die das Spleißen beeinflussen, sowie zwei kleinere Rearrangements [51]), und Marlhens und Kollegen beschrieben zwei weitere Mutationen (eine EinzelnukleotidDeletion [c.1056delA] und eine Missense-Mutation [p.Arg234*] [52]). Seitdem sind unzählige weitere Varianten entdeckt worden, und nicht von allen ist die Pathogenität bereits geklärt.

Parallel zur Entdeckung neuer Varianten schreitet auch die Klassifizierung der Varianten hinsichtlich ihrer klinischen Bedeutsamkeit voran. 2015 haben das American College of Medical Genetics and Genomics (ACMG) und die Association for Molecular Pathology (AMP) eine Leitlinie herausgegeben, die auch eine Standardterminologie zur Einteilung der Varianten in fünf Kategorien («benigne», «wahrscheinlich benigne», «unklare Signifikanz», «wahrscheinlich pathogen», "pathogen») sowie eine Beschreibung des Vorgehens zur Kategorisierung anhand der verfügbaren Evidenz (z.B. Populations-, Computer-, Funktions- und Segregationsdaten) enthält [53].

Im Januar 2021 sind mehr als 300 Variationen des RPE65-Gens in der ClinVar-Datenbank aufgeführt [54]; hiervon gelten etwa 65 als pathogen, 40 als wahrscheinlich pathogen und 100 von unklarer Signifikanz. Die meisten Varianten (>260) sind Einzelnukleotid-Veränderungen [54]. Hierbei ist zu beachten, dass die in ClinVar enthaltene Stichprobe eine Verzerrung zugunsten von pathogenen Varianten aufweisen könnte, da benigne und wahrscheinlich benigne Varianten eher selten in öffentlichen Datenbanken erfasst werden. Gleichzeitig sind 206 RPE65-Varianten in der Leiden Open Variation (LOV-)Datenbank aufgeführt, von denen 102 als pathogen oder wahrscheinlich pathogen und 29 als von unklarer Signifikanz klassifiziert sind [55]. Der Datensatz zu RPE65 in der Genome Aggregation Database (gnomAD) enthält 120 synonyme Einzelnukleotidvarianten (SNV; single-nucleotide variants), 284 Missense-SNV und 24 SNV, die als «mögliche Funktionsverlustmutationen» gekennzeichnet sind [56].

Die Variantenprävalenz und die Merkmale von RPE65 bei 2240 IRD-Patienten wurden in einem nach Clinical Laboratory Improvement Amendments zertifizierten Labor in den USA untersucht [39]. Bei 18 Patienten (0,8\%) war die Erkrankung RPE65-assoziiert, bei 12 davon (6\%) lag mindestens eine Funktionsverlustmutation vor. Unter den 35 in dieser Studie identifizierten Varianten waren zwei (5,7\%) Kopienzahlvarianten (CNV; eine Einzel-ExonDeletion und eine Deletion des gesamten RPE65-Gens) [39]. Während in älteren Studien keine Korrelationen zwischen bestimmten RPE65-Genotypen und dem Phänotyp [57] oder dem klinischen Verlauf [58] gefunden wurde, deutet eine jüngst erschienene Studie darauf hin, dass ein Zusammenhang zwischen dem Mutationstyp und dem Lebensalter bei Krankheitsbeginn besteht [59]. Patienten mit zwei Missense-Allelen hatten einen späteren Krankheitsbeginn ( $\geq 1 \mathrm{Jahr}$ ) als solche mit einer oder zwei trunkierenden Varianten ( $<1$ Jahr; Log-Rank-Test: $p<0,05)$ [59].

\section{Beurteilung von Varianten unklarer Signifikanz}

Varianten von unklarer Signifikanz (VUS) stellen eine große Herausforderung dar, wenn es darum geht festzustellen, wer ein Kandidat für eine Gentherapie bei RPE65-IRD ist [48]. Mahajan et al. stellten jedoch keine Korrelation zwischen dem VariantenSubtyp bzw. ACMG-Klassifikation einerseits und dem Therapieansprechen andererseits fest. 7 der 29 Patienten ihrer Fallserie von Patienten mit einer bestätigten genetischen Diagnose biallelischer RPE65-Genvarianten hatten mindestens eine VUS, und alle von ihnen, einschließlich drei Patienten mit zweifacher VUS, sprachen auf ihre Gensupplementierungstherapie an [60].

Die Pathogenität einer VUS im RPE65-Gen lässt sich auf unterschiedlichen Wegen beurteilen. Dazu gehören erweiterte Segregationsstudien, die Beurteilung des Phänotyps, in-silico-Tools zur Vorhersage der Proteinkonservierung und -funktionalität sowie in-vitro-Funktionsstudien. Darüber hinaus kann eine erweiterte gezielte Sequenzierung oder Exom-Sequenzierung durchgeführt werden, um weitere IRD-Gene als Mitursache der Krankheit auszuschließen.

Segregationsstudien bei allen erreichbaren Verwandten können bei der Bestimmung und Neuklassifizierung der Pathogenität von VUS hilfreich sein. In einer erweiterten Segregationsanalyse wurden die VUS p.Phe83Leu und p.Gly187Glu bei Familien mit
118

Kompass Ophthalmol 2021;7:115-123 DOI: $10.1159 / 000519136$ 
IRD gefunden: die erstere bei fünf LCA/EORD-Patienten aus vier nicht verwandten Familien und die letztere bei sieben Patienten aus drei nicht verwandten Familien. Zugleich waren beide Varianten bei Patienten mit LCA/EORD häufiger als bei Patienten mit anderen IRD. Um die Pathogenität dieser Varianten zusätzlich zu bestätigen, konsultierten die Autoren acht Genvarianten-Datenbanken und setzten 16 Computeralgorithmen ein, um die mutmaßlichen Auswirkungen der Varianten auf die Proteinfunktionalität $\mathrm{zu}$ analysieren; 15 identifizierten die beiden Varianten als schädlich. Die Gesamtklassifizierung von p.Phe83Leu und p.Gly187Glu wurde daher von VUS zu «wahrscheinlich pathogen» geändert [61].

In der ACMG-Leitlinie wurde ein für die Krankheit hochgradig spezifischer Phänotyp des Patienten und eine passende Familiengeschichte bei Krankheiten mit monogener Ätiologie als unterstützendes Kriterium für die Klassifizierung pathogener Varianten eingestuft (das PP4-Kriterium) [53]. In der daraufhin von der britischen Association for Clinical Genomic Science veröffentlichten Leitlinie heißt es, dass es in einigen Fällen angemessen sein kann, PP4 in moderatem oder hohem Maße anzuwenden, nachdem eine Beteiligung anderer Gene ausgeschlossen wurde [62]. Im Fall der RPE65-bedingten IRD würde es für Neueinstufung einer VUS in die Kategorie «wahrscheinlich pathogen» sprechen, wenn bei einem Patienten mit einem typischen LCA/ EORD-Phänotyp (wie in der Einleitung beschrieben) ein Compound-heterozygoter Genotyp mit einer VUS festgestellt wird, ohne dass eine alternative genetische Ursache vorliegt; dies gilt insbesondere bei Vorliegen einer entsprechenden Allelsegregation und in-silico-Vorhersage.

In-silico-Instrumente zur Prädiktion der Pathogenität sind in der Regel in die bioinformatischen Pipelines integriert, die bei Genanalysen mittels Next-Generation-Sequenzierung (NGS) zum Einsatz kommen. Bioinformatische Vorhersagemethoden (z.B. Polymorphism Phenotyping v2 (PolyPhen2), Sorting Intolerant From Tolerant (SIFT) oder Deleterious Annotation of genetic variants using Neural Networks (DANN), um nur einige Tools zu nennen) und Modelle sind zwar sinnvoll, spielen aber in der klinischen Praxis eine weniger große Rolle (eine detaillierte Auflistung der Tools ist in Richards et al., 2015 zu finden [53]). Philp und Kollegen haben einen Algorithmus entwickelt und validiert, den sie «estimate of pathogenic probability» (EPP; Schätzung der Pathogenitätswahrscheinlichkeit) getauft haben und der anhand der Prävalenz und Segregation einer VUS sowie ihrer vorhergesagten Auswirkungen auf die Proteinstruktur die die Pathogenität vorhersagen soll [63]. Außerdem haben Iancu und Kollegen kürzlich eine Strategie zur Reklassifizierung von VUS entwickelt, bei der fünf Pathogenitätsprädiktoren berücksichtigt werden; der resultierende Algorithmus vermochte in Validierungsdatensätzen rund 70\% der VUS korrekt als pathogen zu reklassifizieren [64]. Der ACMG-Algorithmus wurde aktualisiert, um neue Erkenntnisse widerzuspiegeln. Die Klassifizierung von VUS ist zweifelsfrei ein dynamischer Prozess, der sich auch künftig mit der Gewinnung neuer Daten weiter verändern wird - entsprechend wichtig ist der Wissensaustausch in diesem Bereich.
In-vitro-Studien werden nur in der Forschung durchgeführt, da sie arbeits- und kostenintensiv sind. In diesen Studien werden die Auswirkungen von Mutationen auf die Abundanz, Lokalisierung und Funktion des Proteins sowie die Auswirkungen von Spleißstellenvarianten untersucht. Kürzlich wurde ein minimales invitro-Sehzyklus-System in 293-F-Zellen zur Beurteilung der Isomerisierungsaktivität von RPE65 [65] in Kombination mit in-vitro-Mutagenese eingesetzt, um sich vor einer Gentherapie der Pathogenität von RPE65-VUS zu vergewissern. Die Untersuchung ergab, dass die Protein-VUS p.Gly104Val und p.Pro467Ser katalytisch inaktiv waren, und tatsächlich unterzogen die Patienten sich der Behandlung und sprachen darauf an [48]. Dieses Beispiel zeigt, dass die Beurteilung der enzymatischen Aktivität in Verbindung mit der in-vitro-Mutagenese von Nutzen sein kann, um die Pathogenität von VUS zu ermitteln.

\section{Schwierige Fälle von RPE65-IRD}

Die RPE65-IRD sind zwar monogene Erkrankungen, doch einige Autoren diskutieren auch die Eventualität einer «Double-Hit»Ätiologie von IRD oder einer den Phänotyp beeinflussenden Wirkung koexistierender Mutationen [58]. Weiterführende Untersuchungen sind erforderlich, um diese Hypothesen zu bestätigen bzw. zu verwerfen und ihre Auswirkungen auf die Anwendungskriterien von Gentherapien zu ergründen.

Um die Phase der mutmaßlichen Compound-heterozygoten Varianten zu bestätigen - sowohl im Hinblick auf die richtige genetische Beratung als auch um entsprechend die Eignung für eine Gentherapie zu bestätigen - ist eine Segregationsanalyse der Eltern des Probanden erforderlich. Einige Patienten scheinen beim ersten Test homozygot für eine RPE65-Mutation zu sein. Wenn der Patient homozygot für eine bekannte Variante ist und der Phänotyp nach einer RPE65-bedingten Ätiologie aussieht, kann eine solche Segregation im Prinzip weniger zwingend notwendig sein. Dennoch wird eine Segregationsanalyse auch bei «homozygoten» Varianten immer empfohlen, um den Genotyp umfassend zu charakterisieren. Der scheinbaren Homozygotie können drei Szenarien zugrunde liegen: Kopienzahlvariationen, die zum Verlust der Heterozygotie führen [39, 66], uniparentale Isodisomie [67] oder echte Homozygotie infolge genetischer Isolate, d.h. beide Elternteile tragen dieselbe Mutation (was wahrscheinlicher ist, wenn isolierte Lokalisationen betroffen sind, wenn Konsanguinität vorliegt oder in Familien wie in [51] beschrieben). Eine weitere Möglichkeit sind Träger von zwei Varianten in cis und nicht in trans, d.h. auf demselben Allel, während das andere Allel vom Wildtyp ist. Solche Patienten sind keine Kandidaten für eine Gentherapie für autosomal-rezessiv vererbte Krankheiten. Segregationsstudien und Haplotyp-Auflösungen mittels Long Reads sind ein Verfahren der Wahl, um genetische Varianten den homologen väterlichen und mütterlichen Chromosomen zuzuordnen [68]. Die vereinten Bemühungen internationaler Expertennetzwerke für IRD-Genetik sowie die regelmäßige Aktualisierung öffentlicher Variantendatenbanken sind hoch zu bewerten und werden wesentlich zur Klassifizierung von VUS sowie zur Interpretation ungeklärter Fälle beitragen. 


\section{Teststrategie}

Gentests sind der einzige Weg, RPE65-IRD sicher zu diagnostizieren und den Patienten für eine Gentherapie zu qualifizieren. Die Komplexität der Gentestung ist zu bewältigen, indem man interdisziplinäre Expertenteams bildet, die sich mit der Genetik von IRD und der Interpretation von Varianten auskennen, um so die bestmögliche Versorgung des Patienten zu gewährleisten. In Italien und hier insbesondere in auf IRD-Genetik spezialisierten Labors umfassen diese Teams in der Regel Fachkräfte für Molekularbiologie, Labortechnik und medizinische Genetik ebenso wie für Ophthalmologie, Bioinformatik, Molekulargenetik und genetische Beratung. Um das pathogene Potenzial neuartiger Gene oder Varianten zu bestätigen, müssen diagnostisches Fachwissen und grundlagenwissenschaftliches Know-how kombiniert werden, um auch in den komplexesten Fällen den Genotyp zu bestimmen. Eine genetische Diagnose zählt zu den Voraussetzungen für die Behandlung, die im aktuellen Konsenspapier der italienischen IRD-Arbeitsgruppe aufgeführt sind [49]. Das in dieser Arbeit beschriebene schrittweise Verfahren ist ein Weg, um die Sensitivität der genetischen Diagnostik zu erhöhen und für die meisten Patienten eine genetische Diagnose stellen zu können wie von Stone et al. 2017 gezeigt [32]. Für umfassende Gentests bei seltenen Augenkrankheiten setzt sich auch das ERN-EYE-Netzwerk ein, das die klinische Notwendigkeit und Relevanz von Gentests bei diesen Erkrankungen betont [69].

Die Wahl der Teststrategie und die Interpretation der Varianten richten sich maßgeblich nach den Informationen, die der anordnende Arzt dem Labor gibt. Der Kliniker muss eine genaue klinische Diagnose und Informationen zum Stammbaum vorlegen. Idealerweise sollten der Anforderung eines Gentests außerdem die allgemeine Anamnese ebenso beigefügt werden wie die ophthalmologische Anamnese einschließlich einer Beschreibung der retinalen und nicht-retinalen klinischen Manifestationen (Anzeichen eines Syndroms), ein Familienstammbaum den betroffenen Familienmitgliedern und dem Vererbungsmuster sowie Angaben zur Blutsverwandtschaft. Mithilfe dieser Informationen lassen sich die richtigen Phänotyp-Genotyp-Korrelationen herstellen und Hinweise auf eine syndromale oder nicht-syndromale Erkrankung erkennen.

Der technische Fortschritt ermöglicht es, bei bis zu 76\% der Patienten mit IRD die zugrunde liegende genetische Ursache zu identifizieren [32]. Gentests für RPE65-IRD können mittels SangerSequenzierung durchgeführt werden, insbesondere wenn keine NGS-Methoden verfügbar sind. Eine umfassende Testung muss jedoch unseres Erachtens auch andere IRD-Gene mit einbeziehen [32]. Dies gilt insbesondere bei Patienten mit VUS sowie bei der Beurteilung der Voraussetzungen für eine Gentherapie [49]. In diesen Fällen wird die erweiterte Panel-Sequenzierung nachdrücklich empfohlen. Um es kurz zu sagen: Zielgerichtete Panelbasierte Methoden sind schnell und kosten wenig, bieten aber im Falle eines negativen Ergebnisses keine alternative genetische Diagnose. Mit der Whole-Exome-Sequenzierung (WES) lassen sich Varianten in allen codierenden Sequenzen erkennen, mit der
Whole-Genome-Sequenzierung (WGS) sogar überall im Genom, wenngleich diese Verfahren mehr kosten und eine längere und schwierigere Analyse erfordern als die Sequenzierung eines ausgewählten Panels von Genen.

Im klinischen Setting muss ein Diagnoselabor zertifiziert und mit modernen Geräten ausgestattet sein und Erfahrung in der Nutzung moderner Technologien (wie NGS, WES, Multiplex Ligation Probe Amplification (MLPA) oder Sanger-Sequenzierung) haben, um die Qualität der Diagnoseberichte zu gewährleisten. Die Wahl der Teststrategie richtet sich außerdem nach der Abwägung zwischen Kosten, Durchsatzzeit, Sequenziertiefe und Datenspeicherkapazitäten. Die gängigsten Verfahren für eine präzise genetische Diagnose umfassen in der Regel ein NGS-Augen-Panel oder die klinische Exom-Sequenzierung (CES; wird auch zur Diagnose anderer Erkrankungen eingesetzt), gefolgt von einer zielgerichteten in-silico-Analyse nur derjenigen Gene, die bekanntermaßen an IRD beteiligt sind. Die restlichen Gene werden nur dann analysiert, wenn das «virtuelle Augen-Panel» keine genetische Diagnose ergibt; hier können dann neue Gene entdeckt werden, die für den IRD-Phänotyp verantwortlich sind. Dieses Vorgehen könnte zugleich auch den Zeitaufwand für die Gentestung verringern, da keine neue Sequenzierung durchgeführt werden muss, sondern die bereits vorhandenen Daten neu analysiert werden.

Die Standard-Durchsatzzeit für zielgerichtete Tests beträgt etwa 3-4 Monate. Bei Patienten, bei denen IRD-spezifische Panels erfolglos analysiert wurden oder bei denen ein atypischer Phänotyp vorliegt, sieht die Strategie die Durchführung einer CES oder WES [70] oder auch CES gefolgt von WES [71] vor. Eine WGS hingegen soll bei Patienten durchgeführt werden, deren Phänotyp einer bestimmten Erkrankung entspricht, bei denen aber keine Mutationen in den Genen nachgewiesen wurden, die zuvor als verantwortlich für diese Erkrankung identifiziert wurden bzw. bei denen die WES keine Auflösung erbracht hat [70, 72]. So ist bei Patienten mit nur einer pathogenen Variante von RPE65 («monoallelisch») und einem überzeugenden klinischen Erscheinungsbild (RP oder LCA/EORD) die Sequenzierung des gesamten Gens gerechtfertigt, um nach CNV oder komplexen strukturellen Varianten, SNV in nicht-codierenden Regionen (UTR, Promotor- und potenzielle regulatorische Elemente) sowie «tief»-intronischen (DI) Varianten zu suchen. Der Anteil der Fälle mit monoallelischer pathogener RPE65-Variante ist jedoch recht gering; dieser Ansatz wird hauptsächlich im Forschungskontext verwendet. Hingegen machen DI-Varianten $2 \%$ der einzigartigen und $4 \%$ der gesamten ABCA4-Genvarianten aus, die an der Pathogenese einer anderen autosomal-rezessiven IRD, der Stargardt-Krankheit, beteiligt sind. Da Mutationen von $A B C A 4$ häufiger sind als von RPE65, ist es wenig überraschend, dass der Beitrag von DI-Varianten zur Pathogenese der Stargardt-Krankheit früher erkannt wurde [73]. Angesichts sinkender Kosten und Durchsatzzeiten der Sequenzierungstechnologien, und wenn die Kapazitäten zur Interpretation der funktionellen Auswirkungen nicht-codierender Varianten steigen, wird die WGS in der IRDDiagnose zur Methode der Wahl auch im klinischen Umfeld werden. Die Bestätigung der Ergebnisse erfolgt weiterhin durch San-
120

Kompass Ophthalmol 2021;7:115-123 DOI: $10.1159 / 000519136$ 
Abb. 1. Vorgeschlagenes Vorgehen bei der genetischen Diagnostik im Überblick *Wenn benigne oder wahrscheinlich benigne Varianten gefunden werden, sollte die Suche nach der/den für den Phänotyp verantwortlichen Mutation(en) auf andere Gene erweitert werden. CES: klinische ExomSequenzierung; MLPA: multiplexe ligationsabhängige Sondenamplifikation; NGS: Next-GenerationSequenzierung; VUS: Variante unklarer Signifikanz; WES: Whole-Exome-Sequenzierung; WGS: WholeGenome-Sequenzierung.

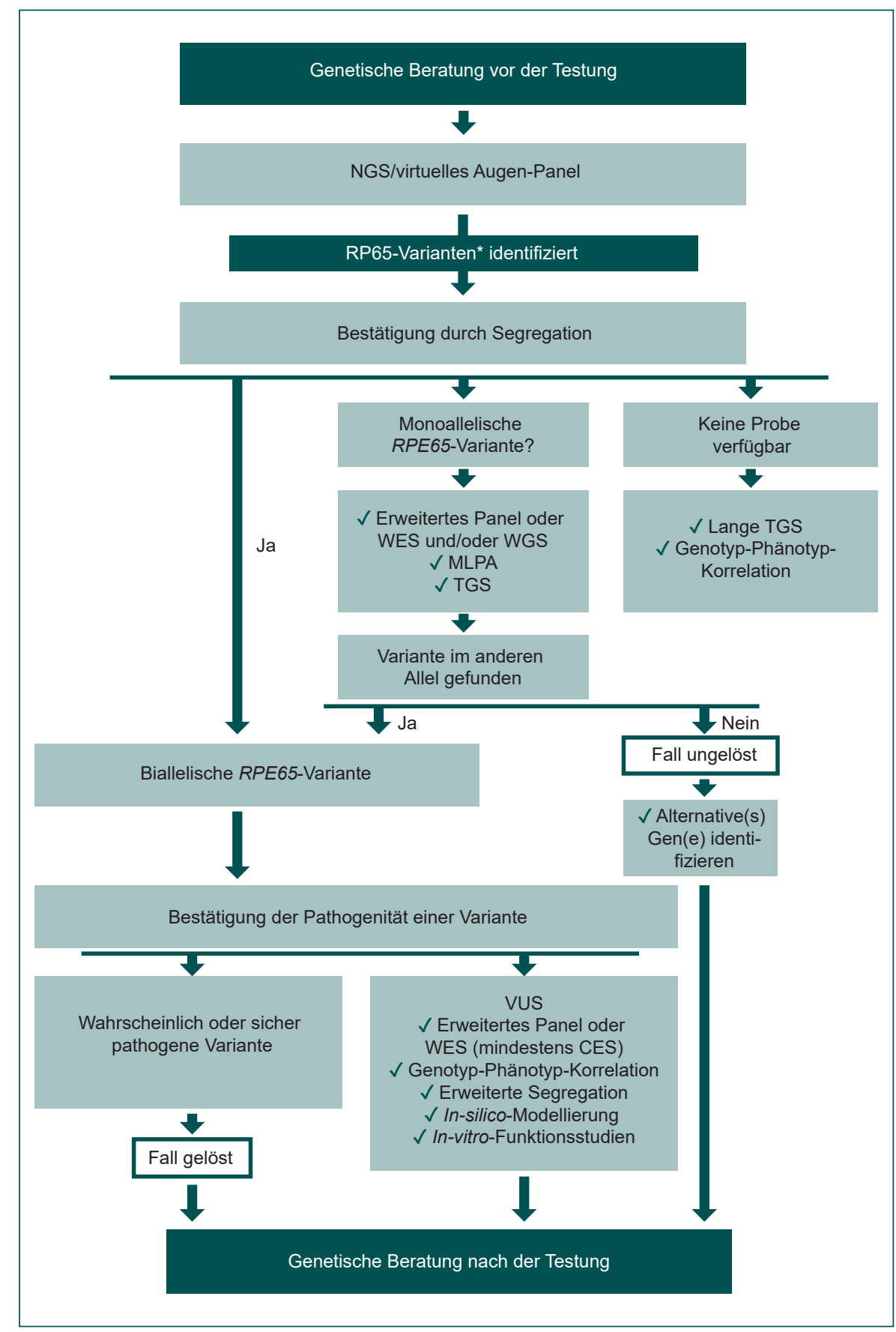

ger-Sequenzierung. Die EVICR.net-Erhebung ergab, dass die meisten befragten Zentren in Europa klinische Tests für die Diagnose von IRD einsetzen; nur wenige kombinieren klinische und wissenschaftliche Ansätze. Die meistverwendeten Methoden waren IRD-spezifische Genpanels (67\%), WES (49\%) und diagnosegeleitete Sanger-Sequenzierung (41\%). Derzeit werden in 69\% der befragten EVICR.net-Zentren $41-80 \%$ der jeweiligen IRD-Patienten genetisch aufgelöst; nur 5\% der Zentren gelangen bei $81-$ $100 \%$ ihrer IRD-Patienten zu einer genetischen Diagnose [74].

Ein Nachteil von NGS-Panels liegt darin, dass sich damit in der Regel keine CNV bestimmen lassen; hierfür soll eine MLPA-Ana- lyse durchgeführt werden, insbesondere wenn eine erweiterte Segregationsanalyse nicht möglich ist (z.B. wenn der untersuchte Patient ein adoptiertes Einzelkind ist). In einer Studie mit 677 Personen mit Netzhautdystrophie wurden in den NGS-Daten vorliegende Hinweise auf eine Deletion oder Duplikation mittels MLPA, qPCR oder Chromosomen-Mikroarray überprüft [75].

Die Segregationsanalyse ist ein grundlegendes Element der genetischen Teststrategie, wie bereits im Abschnitt über Sequenzvarianten beschrieben. Daher ist es wichtig, zum Zeitpunkt der genetischen Untersuchung eines Patienten Proben auch von seinen Eltern zu gewinnen. Die Vorab-Analyse von drei Proben (Trio- 
Analyse) statt einer erhöht zwar die Testkosten, könnte bei sinkenden Kosten aber zu einem Standardverfahren werden. In Abbildung 1 ist ein Vorschlag für das gendiagnostische Vorgehen bei RPE65-IRD zusammengefasst.

Mit der Einführung der dritten Generation der Sequenzierungstechnologie (TGS; third-generation sequencing), die auf der Erzeugung langer Reads basiert, wurde die routinemäßige Auslesung von $>10 \mathrm{~kb}$ möglich. Zwei Technologien spielen hier auf dem Markt bereits herausragende Rollen: die Einzelmolekül-Echtzeitsequenzierung, die als PacBio (von Pacific Biosciences) bezeichnet wird, und die Nanopore-Sequenzierung (Oxford Nanopore Sequencing) [76]. Während das PacBio-Verfahren auf der Erfassung von Fluoreszenzereignissen basiert, die dem Einbau von Einzelnukleotiden entsprechen [77], misst die Nanopore-Sequenzierung Ionenstromänderungen, wenn ein natives einzelsträngiges Nukleinsäuremolekül eine Nanopore passiert [78]. Lange Reads erfordern geeignete Analysewerkzeuge; hierfür wurde kürzlich eine Datenbank angelegt [76]. TGS ist besonders geeignet für Studien zur Genomzusammensetzung und für die Identifizierung von Strukturvarianten (d.h. Mutationen, die mehr als 50 Basenpaare betreffen), wie von Xia und Zhou 2020 beschrieben [79]). Im Kontext der Gentestung bei IRD wird die TGS dazu beitragen, Waisen-Fälle (ohne Segregationsdaten) zu entschlüsseln oder in den oben beschriebenen Fällen von scheinbarer Homozygotie die cis/trans-Allelphase zu erforschen.

Künstliche Intelligenz (KI) könnte künftig den Prozess der Gentestung beschleunigen, die Genauigkeit der Vorhersage der Pathogenität verbessern und den Anteil gelöster genetischen Diagnosen erhöhen. KI-Ansätze in der klinischen Genomik richten sich auf Aufgaben, die mit herkömmlichen Methoden zeitaufwendig und fehleranfällig sind. Aufgaben wie die korrekte Detektion und Klassifikation von Varianten, Genom-Annotation, funktionelle Modellierung und Feststellung der Korrespondenz von Phänotyp und Genotyp - oder in Zukunft vielleicht auch eine Genotyp-Phänotyp-Vorhersage allein anhand von genomischen Daten - werden zunehmend mit Hilfe von KI erledigt werden [80]. Bisher gelang mit tiefen neuronalen Netzen bereits die Vorhersage des IRD-verursachenden Gens bei Makuladystrophie infolge von ABCA4- und RP1L1-Aberrationen im Vergleich zu RP infolge von EYS-Aberrationen und zu gesunden Probanden mit Daten aus der optischen Kohärenztomographie im Spektralbereich [34, 81]. Eine Steigerung der diagnostischen Ausbeute wird letztlich ermöglichen, mehr Patienten in klinische Studien aufzunehmen, welche wiederum zur Zulassung neuartiger Therapieoptionen führen.

\section{Rolle der genetischen Beratung}

Genetische Beratung wird definiert als «Prozess, bei dem Menschen darin unterstützt werden, die medizinischen, psychischen und familiären Implikationen des genetischen Anteils einer Krankheit zu verstehen und sich darauf einzustellen» [82]. Wichtig ist hierbei, dass die genetische Beratung neben der Aufklärung der Patienten über die genetischen Aspekte ihrer Erkrankung auch Elemente der Psychotherapie enthält [83]. Die genetische Beratung ist offenbar wirksam im Hinblick darauf, die Patienten über ihre Krankheit zu informieren und ihr Gefühl der Kontrolle über ihre Situation ebenso zu stärken wie ihre Kompetenz in der Einschätzung von und dem Umgang mit Risiken [84].

Bei Patienten mit IRD profitiert eine genetische Beratung sowohl von vorhandener Erfahrung mit Augenkrankheiten als auch eingehender Kenntnis der genetischen Aspekte. Daher wird dringend empfohlen, dass genetische Berater entweder selbst über Erfahrung in der Augengenetik verfügen oder in interdisziplinären Teams mit Fachkompetenz auf diesem Gebiet arbeiten; insbesondere ist anzuraten, dass ein Augenarzt und ein medizinischer Genetiker Beratungsgespräche gemeinsam durchführen [74].

Bei der genetischen Beratung, die vor der Testung angesetzt wird, müssen die Patienten ihre Einwilligung erteilen, nachdem sie aufgeklärt wurden und sich umfassend informiert haben, insbesondere über das Spektrum der möglichen Ergebnisse, die Bedeutung und die Beschränkungen der Tests (z.B. dass es bei Tests, die mehrere Gene untersuchen, nicht für alle Varianten eine eindeutige Interpretation gibt und dass das Testergebnis oder seine Bedeutung unklar sein können) sowie logistische Herausforderungen und Wartezeiten. Auch die Implikationen für die Familie müssen erläutert werden, unter anderem die Aspekte Vererbungsmuster und Möglichkeiten der Pränataldiagnostik. Nicht zuletzt müssen die Berater das Thema Zufallsbefunde besprechen, insbesondere wenn eine Untersuchung des ganzen Exoms/Genoms geplant ist. Bei NGS-Untersuchung eines Panels in silico (virtuell) besteht die Möglichkeit solcher Zufallsbefunde nicht, da nur bekannte IRDGene getestet werden. Wenn jedoch eine WES oder WGS durchgeführt wird und einen Zufallsbefund ergibt, hat der genetische Berater die äußerst wichtige Aufgabe, die Konsequenzen daraus mit dem Patienten und seiner Familie zu besprechen. Patienten können vielerlei Vorbehalte gegen Gentests haben, zum Beispiel ethische Bedenken, Vaterschaftsfragen, der Unwille, Familienmitglieder mit hineinzuziehen sowie mangelnde Informiertheit. Manche Patienten befürchten gesagt zu bekommen, dass es für sie keine Heilung gibt (nicht bei RPE65-IRDs); Eltern können irrationale Schuldgefühle haben, weil sie eine Krankheit weitergegeben haben, und auch die Angst vor einer genetischen Krankheit bzw. Realitätsverweigerung spielen eine Rolle.

Die Kommunikation mit den Patienten und ihren Angehörigen ist eine schwierige, aber überaus wichtige Aufgabe. Das medizinische Fachpersonal muss sich ausreichend Zeit für die genetische Beratung nehmen (eine Sitzung dauert im Durchschnitt etwa 45 Minuten), und die Patienten müssen das Gefühl haben, dass sie über ihre Erwartungen und Ängste sprechen dürfen und dass das Fachpersonal bei Bedarf für sie da ist. Manchmal braucht es auch mehrere Gesprächstermine, bis die Patienten das Gefühl haben, dass sie ihre Situation verstehen und im Griff haben und fundierte Entscheidungen treffen können. Da die RPE65-IRDPatienten oft sehr kleine Kinder sind, kann das Gespräch mit den Eltern/Betreuungspersonen für alle Beteiligten sehr belastend sein. 
Wenn die Testergebnisse vorliegen, sollte sich der Schwerpunkt der genetischen Beratung auf der Interpretation dieser Ergebnisse liegen, z.B. ob es für den Phänotyp relevante oder nicht relevante Varianten sind, ob eine VUS oder eine unerwartete Diagnose gefunden wurden; im Falle eines unklaren Ergebnisses kann außerdem auf die Notwendigkeit weiterer Analysen und die Einbeziehung von Angehörigen eingegangen werden. In der genetischen Beratung nach dem Test wird auch über das Krankheitsmanagement (interdisziplinäre Versorgung von Syndrompatienten) und therapeutische Optionen gesprochen, ebenso über eine etwaige Teilnahme an einer klinischen Studie oder Testung weiterer Familienmitglieder. Wenn zusätzliche genetische Untersuchungen erforderlich sind, muss die erneute Wartezeit auf eine genetische Diagnose thematisiert werden. Da eine Segregationsanalyse oft ein Schlüsselelement der molekularen Diagnose ist, bedeuten Patienten mit fortgeschrittener IRD und ohne Familienangehörige eine besondere Herausforderung für die genetische Testung und Beratung dar.

\section{Schlussfolgerungen}

Es gibt heute eine Gentherapie zur Behandlung von RPE65-IRD. Therapien für andere Genotypen befinden sich in der Entwicklung; klinische Studien zur Sicherheit und Wirksamkeit sind bereits eingeleitet [85]. Nur Patienten mit einer ausreichenden Anzahl lebensfähiger Netzhautzellen können in vollem Umfang von der Therapie profitieren. Deshalb muss eine Strategie für rechtzeitige und wirksame Gentests implementiert werden, um passende Kandidaten für eine Gentherapie durch ein umfassendes schrittweises Verfahren zu identifizieren, sodass kein Patient ohne genetische Diagnose bleibt. Um die bestmöglichen Outcomes für diese Patienten zu erzielen, sollten außerdem genetische Beratungsprogramme geschaffen werden. Da Phänotyp und Genotyp oft nicht miteinander korrelieren, sollten interdisziplinäre Teams aus den Bereichen Klinik, Genetik, Beratung, Labordiagnostik und Grundlagenforschung gebildet werden, die die $\mathrm{Zu}$ sammenhänge zwischen ihren Perspektiven untersuchen und die molekularen/pathogenen Mechanismen jeder Variante ergründen, um VUS zutreffend zu klassifizieren. Häufige Interaktion, kontinuierlicher Austausch und Kooperation optimieren die Patientenversorgung und maximieren den Nutzen der verfügbaren Therapien für die Patienten.

\section{Beiträge der einzelnen Autoren}

M.A. konzipierte die Arbeit, berief ein Redaktionsgremium ein und entwarf die Gliederung; S.B. und M.S. konzipierten, strukturierten und überwachten die Verfassung des Manuskripts. Alle Autoren trugen Erkenntnisse zur Ausarbeitung des Manuskripts bei, überprüften seinen intellektuellen Gehalt und gaben die finale Fassung zur Veröffentlichung frei. Alle Autoren haben das Manuskript in der veröffentlichten Fassung gelesen und freigegeben.

\section{Finanzierung}

Die Autoren erhielten keine externen Mittel für diese wissenschaftliche Arbeit.

\section{Danksagungen}

Wir danken Georgii Filatov und Alicja M. Gruszka von Springer Healthcare Communications für die Erstellung der Gliederung (GF) und das Verfassen des Manuskripts (AMG) sowie Vincenza Vinaccia von Novartis Pharmaceuticals Italy für die kritische Durchsicht des Manuskripts. Die redaktionelle Unterstützung wurde von Novartis Farma finanziert.

\section{Disclosure Statement}

M.A. und N.F. sind Angestellte von Novartis Pharmaceuticals, Italy und Region Europe. I.P., P.C., M.K., I.D.R., V.M., M.S. und S.B. waren als Experten in Beiräten für Novartis Pharmaceuticals, Italy und Region Europe tätig. S.B. hat Seminarleitungs-Honorare von Novartis Pharmaceuticals, Italy und Region Europe erhalten. G.S. erklärt, dass keine Interessenkonflikte bestehen.

\section{Lizenzangabe}

Aoun M, Passerini I, Chiurazzi P, Karali M, De Rienzo I, Sartor G, Murro V, Filimonova N, Seri M, Banfi S. Inherited Retinal Diseases Due to RPE65 Variants: From Genetic Diagnostic Management to Therapy. Int J Mol Sci. 2021;22(13):7207. DOI: 10.3390/ijms22137207. ${ }^{\circ} 2021$ The Authors. Licensee MDPI, Basel, Switzerland (Übersetzung; Institutional Review Board Statement, Informed Consent Statement und Data Availability Statement gekürzt), lizensiert unter CC BY 4.0 (https://creativecommons.org/licenses/by/4.0/deed.de).

\section{Literatur}

Die Literatur ist unter www.karger.com/doi/10.1159/000519136 abrufbar. 